\title{
The White Dwarf Age of NGC $2477^{1}$
}

\author{
Elizabeth J. Jeffery \\ Space Telescope Science Institute, 3700 San Martin Drive, Baltimore, MD 21218, USA \\ jeffery@stsci.edu \\ Ted von Hippel \\ Department of Physics and Astronomy, Siena College, Loudonville, NY, USA \\ Steven DeGennaro \\ Department of Astronomy, University of Texas at Austin, Austin, TX, USA \\ David A. van Dyk \\ Department of Statistics, The University of California, Irvine, CA, USA \\ Nathan Stein \\ Department of Statistics, Harvard University, Cambridge, MA, USA \\ William H. Jefferys \\ Department of Astronomy, The University of Texas at Austin, Austin, TX, USA \\ Department of Mathematics and Statistics, The University of Vermont, Burlington, VT, USA
}

\begin{abstract}
We present deep photometric observations of the open cluster NGC 2477 using $H S T / W F P C 2$. By identifying seven cluster white dwarf candidates, we present an analysis of the white dwarf age of this cluster, using both the traditional method of fitting isochrones to the white dwarf cooling sequence, and by employing a new Bayesian statistical technique that has been developed by our group. This new method performs an objective, simultaneous model fit of the cluster and stellar parameters (namely age, metallicity, distance, reddening, as well as individual stellar masses, mass ratios, and cluster membership) to the photometry. Based on this analysis, we measure a white dwarf age of $1.035 \pm 0.054 \pm 0.087$ Gyr (uncertainties represent the goodness of model fits and discrepancy among models, respectively), in good agreement with the cluster's main sequence turnoff age. This work is part of our ongoing work to calibrate main sequence turnoff and white dwarf ages using open clusters, and to improve the precision of cluster ages to the $\sim 5 \%$ level.
\end{abstract}


Subject headings: open clusters and associations: individual (NGC 2477), - white dwarfs

\section{Introduction}

Age is one of the most important quantities in astronomy and is essential to a number of astrophysically interesting problems. From the fundamental questions of the formation of the universe to the creation of planets, knowing and understanding the ages of celestial objects is important. There are currently two main ways to measure the age of stellar populations: mainsequence (MS) evolution theory (via cluster isochrones) and white dwarf (WD) cooling theory. These two methods are especially important in understanding the ages of the constituents of our own Milky Way. Ages determined from the MS turnoffs (MSTOs) of many globular clusters have long been used to provide the age of the Galactic halo (e.g., Chaboyer et al. 1996), while WD cooling ages of field WDs provide the most reliable age of the Galactic disk (Winget et al. 1987; Oswalt et al. 1996; Leggett et al. 1998; Knox et al. 1999).

However, before ages determined by these two techniques can be meaningfully compared and the full picture of Galaxy formation understood, they must be calibrated to the same absolute scale. The only way to empirically perform this calibration is to measure and compare the ages determined from both methods in a single-age, single-metallicity stellar population. Open clusters provide the ideal environment for such a calibration. In addition to a calibration of these two chronometers, measuring the age of a single cluster both ways allows us to compare WD theory with MS theory (and vice versa), providing an excellent opportunity for the refinement of both.

Using WDs as the means to measure the age of a stellar population is conceptually straightforward. There are no appreciable internal energy sources in a WD (such as nuclear fusion), so it shines because it is hot, and as time passes the star cools. Mestel (1952) first showed that there is a relatively simple relationship between the cooling time (i.e., age) of a WD and its brightness. When considered in a simple, conceptual way, this means that if we can measure the brightness of the faintest WDs in a stellar population, we can measure the population's age. Although this simplified view of a WD can be helpful in conceptualizing the determination of a WD cooling age, in practice, WD cooling models are more complicated than this, and measuring an accurate WD age requires other information, including the WDs mass.

The first studies to apply this technique in open clusters were done by Claver (1995) and von Hippel et al. (1995). Later studies (von Hippel \& Gilmore 2000; von Hippel 2001; Claver et al. 2001; DeGennaro et al. 2009; Bellini et al. 2010) showed good agreement in WD ages and MS ages for clusters up to 4 Gyr. A summary of these studies and techniques has been recently presented

\footnotetext{
${ }^{1}$ Based on observations made with the NASA/ESA Hubble Space Telescope, obtained at the Space Telescope Science Institute, which is operated by the Association of Universities for Research in Astronomy, Incorporated, under NASA contract NAS5-26555.
} 
by von Hippel (2005). This technique has also been applied to two globular clusters (M4: Hansen et al. 2002; NGC 6397: Hansen et al. 2007), and a third (47 Tuc, Richer 2010) is underway. These deep observations of the faintest WDs in these globular clusters represents a remarkable triumph for observational astronomy.

While measuring the age of a stellar population with the population's WDs seems simple, WD cooling models are complicated. Substantial work has been done to better understand them (e.g., Montgomery et al. 1999, Salaris et al. 2000), but many puzzles remain, especially among the coolest WDs (e.g., Kilic et al. 2006). This is one of the reasons the studies of WD in star clusters is so important. Studies of WDs in globular clusters allow us to study these coolest WDs in a more controlled, uniform population. Open clusters are also important, especially for the calibration of the WD and MSTO ages, as it allows us to gradually push up the calibration limit in order to better understand the WD cooling physics. This paper adds to the ongoing effort of our and other groups to study WDs in clusters. Our work complements the immense efforts that have gone into the studies of the WDs in the great globular clusters.

An illustration of the need for continuing studies of WDs in open clusters and our incomplete understanding of stellar evolution and the formation of WDs is the recent case of the old, metal-rich open cluster NGC 6791. This cluster's faint WDs were first observed by Bedin et al. (2005) and initial observations showed a peak in the WD luminosity function (LF) that, when fit with standard models, indicated an age of $2.4 \mathrm{Gyr}$, in stark disagreement with the MSTO age. Subsequent studies have sought an explanation for this. One such explanation was put forth by Hansen (2005), who suggested that the bright peak was due to a large population of helium-core WDs, formed when stars experience excessive mass loss (due to the cluster's high metallicity) along the red giant branch (RGB), thereby skipping the helium flash and prematurely becoming WDs. Kalirai et al. (2007) used spectroscopic observations to confirm the existence of such a population. Additionally, a second, fainter peak in the WDLF of NGC 6791 was discovered with deeper observations (Bedin et al. 2008b), as Hansen (2005) also predicted. However, Van Loon et al. (2008), using Spitzer observations, found no evidence of excessive circumstellar dust around the cluster RGB stars, indicating that these stars were not experiencing extreme mass loss. Other groups have also sought to explain the strange bimodal WDLF of this cluster, employing scenarios such as high binary fraction (Bedin et al. 2008a), and the gravitational settling of ${ }^{22} \mathrm{Ne}$ in the core causing the cooling rate of old metal-rich WDs to be slower than normal (Deloye \& Bildsten 2002; Garcia-Berro et al. 2009).

The puzzle of NGC 6791 illustrates the need to continue observations of WDs in open clusters. Our goal is to perform the calibration of MSTO and WD ages using open clusters, covering a range of ages and metallicities. Pushing up the limit of calibration gradually will allow us to better understand and improve upon any uncertainties in the WD cooling physics, as well as MSTO physics.

This paper presents a study of the open cluster NGC 2477. We have observed it with the Hubble 
Space Telescope (HST) to obtain photometry of the cluster WDs. This rich cluster is moderately old (Kassis et al. 1997 measured a MSTO age of 1 Gyr), with a distance modulus of 10.61, and differential reddening with an average value of $E(B-V)=0.3$ (Hartwick et al. 1972). It is slightly metal poor, with $[\mathrm{Fe} / \mathrm{H}] \sim-0.14$ (Eigenbrod et al. 2004). We have listed these and several other literature sources for these cluster parameters in Table 1, This cluster was first studied using HST by von Hippel et al. (1995), who found a preliminary WD age of 1.0 Gyr. In a subsequent paper, von Hippel et al. (1996) studied the lower mass MS. In this paper we reanalyze these HST data and incorporate unpublished Cycle 6 data, using updated HST reduction and photometry techniques, new ground-based data for the brighter stars, new stellar evolution models, and our new Bayesian statistical technique.

We have organized this paper as follows: Section 2 outlines our observations and data reduction techniques, as well as presenting the deep color-magnitude diagram (CMD) for this cluster. In Section 3 we measure an age for NGC 2477 by fitting WD isochrones to the candidate cluster WDs. We discuss our new Bayesian algorithm in Section 4. We developed this technique to objectively fit models to CMDs and measure cluster ages to higher precision than previously possible. We then apply this technique to NGC 2477 and discuss the results in Section 5.

\section{Observations and Data Reduction}

Two fields of NGC 2477 were observed with the Wide Field Planetary Camera 2 (WFPC2) aboard HST. Data were taken in two filters, $F 555 W$ and $F 814 W$ (equivalent to broadband $V$ and $I$, respectively). These fields were observed on three days over two cycles: 1994 March 18 (Cycle 4), 1996 March 25, and 1997 March 18 (both Cycle 6). We have listed coordinate information for the two fields in Table 2 and summarized the observations in Table 3 ,

Basic image calibrations, such as bias correction and flat fielding, were performed by the WFPC2 pipeline (Baggett et al. 2002), using the most up-to-date calibration files. We performed the drizzle procedure on the data using a detailed cookbook and IRAF2 script provided by STScI in the HST Dither Handbook (Koekemoer et al. 2002).

Sources for photometry were found and morphologically classified using SExtractor (Bertin \& Arnouts 1996). We employed CCDCAP (Mighell \& Rich 1995) to derive instrumental magnitudes via aperture photometry. To perform aperture photometry, we utilized a stellar aperture of 4 pixels (compare with the FWHM of $\sim 3.0$ pixels for the drizzled images), with a sky annulus of radius 10 pixels $\left(0^{\prime \prime} .5\right)$, with a width of 3 pixels. The size of the stellar aperture was chosen for consistency with the aperture used by Dolphin (2000) when calculating charge transfer efficiency (CTE) corrections. We used these corrections from Dolphin (2000) with the most up-to-date corrections available on

\footnotetext{
${ }^{2}$ IRAF is distributed by the National Optical Astronomy Observatory, which is operated by the Association of Universities for Research in Astronomy, Inc., under cooperative agreement with the National Science Foundation.
} 
his Web site 3 . The corrections we used here were taken from the 2008 October update of his Web site.

The zero points (ZPs) and color terms we used were those determined by Holtzmann et al. (1995) and updated by Dolphin (2000). We used the most up-to-date values (available from Dolphin's Web site) to transform raw, CTE-corrected WFPC2 magnitudes to standard $V$ and $I$ magnitudes. We note that the cluster observations were taken with the gain setting of seven, so the appropriate ZPs were used. We also applied an aperture correction to correct the smaller aperture measurements of the observations to the appropriate size of the standards.

In the left panel of Figure 1, we present the CMD of all the objects in our WFPC2/HST fields, with only objects with magnitude $(V$ and $I)$ errors less than 0.1 plotted. Overplotted in this figure is the cooling track of a $0.6 M_{\odot}$ WD (Wood 1992), assuming a true distance modulus (i.e., $\left.(m-M)_{0}\right)$ of 10.74 and reddening $(E(B-V))$ of 0.23 , Salaris et al. 2004). We note that the average WD mass of this cluster is likely higher than $0.6 M_{\odot}$; however, since this is roughly the average of field WDs (e.g., Kepler et al. 2007), it serves as a good first approximation in helping us locate the position of the WD sequence.

The right panel of Figure 1 combines the $H S T$ data (large black points) with a set of new photometric observations of the upper MS of NGC 2477 (small black and gray points). (The black points are those that lie within 300 arcsec of the cluster center, and are therefore more likely to be cluster members, while the gray points lie outside the cluster core.) The details of these data will be forthcoming in a later paper (E. J. Jeffery et al., in preparation). Briefly, we observed NGC 2477 with the SMARTS 1-m telescope and Y4KCam CCD camerd 4 at Cerro Tololo Inter-American Observatory. The CCD provides a $20 \times 20$ arcmin field of view with a scale of 0.298 arcsec per pixel. Observations were taken with standard $B V I$ filters and achieve a signal-to-noise ratio of 5-10 at $V \sim 19.5$. In the current analysis, the data in $V$ and $I$ will be used in combination with the $H S T$ photometry of the cluster WDs.

Because the CMD of this cluster is somewhat complicated, we have classified it into four main parts, as diagramed in the right panel of Figure 1 Region 1 is the cluster MS. Region 2 is a significant background population that has merited some discussion in the literature (e.g., Momany et al. 2001); some argue that it is part of the Canis Major overdensity system (Bellazzini et al. 2004), while others argue that it is not part of the CMa system (Carroro et al. 2005). Nonetheless, it is well accepted that this background population exists and that it is not associated with NGC 2477. Region 3 includes other field stars, image defects, and background galaxies. And finally, Region 4 is the cluster WD cooling sequence.

Also included in the right panel of Figure 1 are two horizontal dashed lines on the upper MS.

\footnotetext{
http://purcell.as.arizona.edu/wfpc2_calib/

${ }^{4}$ More information about the Small and Moderate Aperture Research Telescope System (SMARTS) can be found at http://www .astro.yale.edu/smarts/
} 
This indicates the region of the MS used in our Bayesian analysis and will discussed further in Section 4.2.

Our observations were taken at multiple epochs (see Table 3), so we investigated the feasibility of measuring proper motions (PMs) in order to clean the CMD. PMs would be extremely useful for separating the cluster members from the field (especially with such a large background field population). The observations for Field 2 were taken on the same day, making measurements of PMs impossible. The observations of Field 1 were taken just two years apart. Dias et al. (2006) measured a PM for this cluster as differing from the field by less than 2 mas year ${ }^{-1}$. Given this, we would expect $\sim 3.5$ mas of movement over the two-year baseline, translating to 0.07 WFPC2 drizzled pixels.

Because we are reanalyzing data taken during Cycle 4 and 6, i.e., taken before we properly understood subpixel offsets and drizzling procedures, the resulting drizzled point spread functions (PSFs) are not as well constructed when compared to current standards, simply because of the way the data were taken. The result is that we cannot calculate the centroids to the precision required to measure the PM of this cluster. Fortunately, we can use morphological information to reject many of the background galaxies (see Section 3), allowing us to proceed in our analysis without PM information.

\section{Fitting White Dwarf Isochrones}

Despite contamination in the region of the cluster WDs being minimal (see Figure 1), stargalaxy separation was still an important point of concern. Because of small shifts among the images input to the drizzle algorithm, SExtractor had a difficult time distinguishing between stars and galaxies on the drizzled images. To mitigate this problem, we ran SExtractor on images that were a simple combination of individual images (using the IRAF IMCOMBINE task, rather than drizzle), and matched the object classification with the master (drizzled) photometry values. We present a plot of stellarity versus magnitude in Figure 2, The so-called "stellarity index" ranges from 0 (galaxies) to 1 (stars). All sources with a stellarity index less than 0.78 were automatically

rejected. This threshold was chosen based on results from von Hippel \& Gilmore (2000), although the final WD candidates all had stellarity indices greater than 0.90 (i.e., they are definitely stars). The stellarity values provided the first cut toward isolating a clean stellar sample of cluster WDs candidates.

After the stellarity cut, nine possible WD candidates remained. We plot these in Figure 3 , a zoomed region of the CMD around the region of the WDs. We visually inspected each object on the original images to confirm the stellar nature of each one and to exclude any image defects (hot pixels, diffraction spikes, etc) or other non-stellar objects. In Figure 4, we display the portion of the image used for visual inspection with each of these nine possible WD candidates indicated. This examination confirms that objects 1-7 are stellar, while 8 and 9 are likely image defects, found 
in the noisy vignetted edge region of the CCD. Because of this, these two objects were discarded from further analysis. In our first analysis, using standard WD isochrone fitting, we assume that the stellar objects in the WD region are primarily cluster WDs. We relax this assumption in our full Bayesian analysis, as mentioned below.

As an important consistency check, we calculated how many WDs we would expect to find, based on the number of stars we observe on the MS. To do this calculation, we first count the number of MS stars in a particular magnitude range. (We chose bright MS stars, so as to avoid the complications of incompleteness.) We then simulate a cluster with the previously published age and metallicity of NGC 2477, incorporating a Miller \& Scalo initial mass function (IMF), MS evolution timescale models of Dotter et al. (2008), the initial-final mass relation of Weidemann (2000), WD cooling timescales of Wood (1992), and WD atmospheres color from Bergeron et al. (1995). Because this process can be somewhat stochastic, we produced 1000 clusters in this manner and selected only those clusters with a number of MS stars in the same magnitude bin equal to that determined for NGC 2477, totaling 165 simulated clusters. From these 165 clusters, we calculated the average and standard deviation of the number of WDs produced in each instance to be $6.5 \pm$ 2.4. This is a good estimate of the number of WDs we expect to find. Our finding of seven WD candidates falls within the expected number.

We also expect the number of field WDs or unresolved quasars in this region to be small. To estimate the total number of background quasars, we first take the observed number denisty of these objects from recent Sloan Digital Sky Survey observations (e.g., Richards et al. 2009) and use a quasar LF (e.g., Richards et al. 2006) to extend this number density from their limiting magnitude to that of our cluster WD terminus. By then scaling this number density to the field of view of our observations, we expect to find less than one quasar in our field. We therefore do not expect background quasars to be a problem and proceed with our analysis.

In many cases when observing the faintest members of a cluster, observational incompleteness becomes an important issue. von Hippel et al. (1996) calculated the incompleteness of Field 1 to be $\sim 98 \%$ at the level of the coolest WD candidates (see their Figure 1). Field 2 was taken with similar exposure times, so we can assume the completeness to be similar. Because of this high completeness, the data do not need to be corrected for observational incompleteness.

We fit isochrones to the candidate WDs to estimate the WD age for NGC 2477. In Figure 3 , we overplot several Wood (1992) isochrones for ages of 0.5, 1.0, and 1.5 Gyr, as labeled. Objects 1 through 7 are our WD candidates. We have listed the photometry and coordinate positions for these stars in Table 4. This demonstrates a best fit to the terminus of the WD cooling sequence with the 1.0 Gyr isochrone. This is in good agreement with the MSTO age (Kassis et al. 1997). We will discuss error analysis in a later section. 


\section{A Bayesian Approach to Measuring Cluster WD Ages}

The method used in the previous section for determining cluster ages (that is, fitting isochrones to the WD cooling sequence) has served us well for years. However, we desire a more objective approach to fitting models to the data, as well as to increase the precision of the fit. Our goal is to improve the precision of both MSTO and WD ages to 5\%. Because no qualitative gains are likely to be made in the near future in the precision of cluster photometry, stellar abundances, or cluster distances, our best hope of achieving this high age precision is from improved models and improved statistical fitting procedures.

In this section, we will discuss the application of a powerful new Bayesian technique we have developed to determine cluster ages with higher precision. We give a brief overview of the technique and then apply it to the data set discussed in Section 2 to measure the WD age of NGC 2477 from the cluster WDs.

\subsection{Overview of the Technique}

Our Bayesian technique derives posterior distributions for various cluster and stellar parameters by utilizing Bayesian analysis methods. These parameters include age, distance modulus, metallicity, and line-of-site reddening, as well as individual stellar masses, mass ratios, and cluster memberships probabilities. For a thorough and in depth discussion of the technique and its first applications, we refer the reader to von Hippel et al. (2006), Jeffery et al. (2007), DeGennaro et al. (2009) and van Dyk et al. (2009). However, for the convenience of the reader, we provide a short overview of the technique here.

Our Bayesian algorithm aims to fit a cluster evolution model using clear statistical principles. In our current analysis, the cluster evolution model is as follows. It incorporates a Miller \& Scalo (1979) IMF, one of three available MS evolution timescale models (namely, the models of Dotter et al. 2008 (DSED), Yi et al. 2001 (YY), and Girardi et al. 2000), the initial (MS) mass-final (WD) mass relation of Weidemann (2000), WD cooling timescales of Wood (1992), and WD atmospheres colors from Bergeron et al. (1995). In our current analysis, we will compare and discuss the results of using each of the three MS evolution timescale models, while the other model ingredients are held fixed.

Bayes' theorem is at the heart of Bayesian statistics. It states that the posterior probability distribution of the parameters of our model (e.g., cluster age) is proportional to the product of the prior probability distribution and the likelihood function. The prior probability distribution incorporates any information from outside the data, including the cluster-wide parameters age, metallicity, distance, and reddening, and individual stellar parameters such as cluster membership probability (if available from either PMs or radial velocity measurements), mass determinations, and the mass ratio of any unresolved companions. The likelihood function compares the predicted 
photometry for each star (via our cluster evolution model) with the observed photometry assuming known errors in the latter.

Because of the high dimensionality and complex nature of these distributions, the equations cannot be manipulated analytically. We use a Markov chain Monte Carlo (MCMC) technique to sample the posterior distributions of the different cluster-wide parameters, as well as individual stellar quantities such as the mass of each star, mass ratio of any unresolved binary companions, and membership probability. The convergent MCMC chain provides a (correlated) sample from the posterior distributions of the corresponding quantities, and can be used to compute means and intervals as parameter estimates and error bars.

We wish to note that the precision of the parameters discussed in the following sections is internal precision, rather than external accuracy. Our technique objectively determines the best fit of the model to the data, and the goodness of that fit; it cannot assess the physical accuracy of the model itself. For example, a change in the initial-final mass relation could change our results systematically, but the internal precision would still be high. A better understanding of such external modeling issues comes as we intercompare results from multiple model sets, as we begin to do here.

\subsection{Input Data}

For the application of the Bayesian technique 5 , we use the photometry of the seven WD candidates (see Table 4) and ground based photometry of the cluster MS (taken from E.J. Jeffery et al. in preparation, as discussed in Section 21). Only a small portion of the MS is used; for this cluster we used the MS between $V=14.5$ to 15.5. Assuming a reddened distance modulus of 11.45 for this cluster, which translates to an absolute magnitude of $3.05-4.056$, or a mass limit of 1.16 $1.40 M_{\odot}$, we have indicated these limits with the dashed horizontal lines in Figure 1, (We note that everything outside the dashed horizontal lines, except the seven WD candidates, was discarded from the Bayesian analysis.) The upper MS magnitude cutoff was imposed to remove the entire turn off region, in order to derive age information from the WDs alone.

A lower MS limit was imposed to control the amount of MS to be included in each run. The MS fit provides the primary constraint on cluster metallicity, distance, and reddening. While models tend to fit the upper MS well, most do a poor job at fitting the lower MS. Because the MCMC fit is predicated upon the cluster evolution model, the poor fit of the model to the data can result in poor fits of certain cluster parameters. (This specific issue was explored extensively by DeGennaro et al. 2009.) To bypass this problem, we only use the upper MS (below the MSTO) where the

\footnotetext{
${ }^{5}$ For ease, from this point forward we will refer to the Bayesian algorithm simply as "MCMC."

${ }^{6}$ The exact limits on the MS are relatively unimportant, as long as the criteria of the upper and lower limits, discussed in this section, are met.
} 
models better fit the observed shape of the cluster MS. For this reason, the MS observed from the deep HST photometry was not used. Rather, MCMC was given the WD photometry from HST observations and a MS from the ground based data from E.J. Jeffery et al. (in preparation).

\subsection{Running MCMC}

For each of the three MS stellar model sets, we ran a total of twelve separate MCMC chains. Each chain was set to sample for $2 \times 10^{6}$ iterations, reading out every 100th iteration, until 20,000 values of the posterior distribution have been saved. Prior to each run was a burn-in period. During this burn-in period, which totaled 130,000 samples per chain, the MCMC chain was allowed to stabilize, and various correlations were calculated (see DeGennaro et al. 2009 for more details). This allows for more efficient sampling during the MCMC run. We note that all statistics were calculated after the burn-in.

We initiated each of the chains at the prior means of metallicity, distance and reddening (see Table 5). We used a flat prior on $\log ($ age). To check for sensitivity to the starting value of this parameter of primary interest, as well as to look for different modes in the posterior distribution (i.e., competing best/good fits), we started chains at three different starting values for age, namely $\log ($ age $)=8.9,9.0$, and 9.1. Starting values for the masses were compiled by creating an isochrone at the starting value of the cluster parameters and computing the mass for each star that results in the best match between its observed colors and magnitudes and the created isochrone. Because no additional information was available (from radial velocity data or PMs), we set the starting values for the mass ratios to 0.1 and cluster membership priors to 0.5 . All of this results in three chains for each MS model, for a total of nine chains. As we describe below, this entire scenario was replicated four times using different random seeds, or twelve chains for each MS model, for a grand total of 36 MCMC chains.

We compared the results for the three starting $\log$ (age) values for each model, and in each case, MCMC consistently found the same location of the posterior distribution. This demonstrates both the robustness of the algorithm on determining the posterior distribution, as well as indicate the plausibility that no other modes in the posterior distribution exist in this region of parameter space. We plot this result in Figure 5 for the DSED models, but note similar results for both the YY and Girardi models. In this plot, we show the sampling histories of these runs as histograms. Each panel is a different cluster parameter (as labeled). The three line styles represent the three start values of $\log$ (age): solid (8.9), dashed (9.0), and dotted (9.1). Clearly, MCMC finds the same posterior distribution, regardless of the start value.

For each combination of stellar evolution model and starting value for log(age), we set MCMC to run four times, using four different initial seeds. Each time, the MCMC chain stabilized and sampled well. We demonstrate this in Figure 6. This represents multiple runs starting at log(age) = 9.0, using the DSED models, although results were similar for both the YY and Girardi models, as 
well as for other log(age) start values. This demonstrates that the posterior distribution is located and well sampled, regardless of the initial random seed.

\section{Results}

Once we had the full posterior distributions, we were able to calculate statistics. We emphasize that the best summary of our analysis is the complete posterior distribution. Because of its high dimensionality (four cluster parameters plus three parameters for each star), we focus on simpler and more familiar summaries that are easier to compute. In particular, we report the average and the standard deviation of the sample MCMC chains combined from individual runs with each of the three starting values for $\log$ (age) and each of the four random seeds. Again, we emphasize that the precision reported here is internal precision.

We compare the values determined by each of the MS evolution model sets we used. In Figure 7, we overplot the full posterior distributions of each model set, a combination of the twelve individual runs for each model set, as discussed in Section 4.3. Colors and line styles consistent with DeGennaro et al. (2009), namely solid purple (Girardi et al. 2000), dotted red (Yi et al. 2001), and dashed blue (Dotter et al. 2008). The average and standard deviation for each model set is listed in Table 6. The DSED and Girardi values agree well on the age, while the YY models give a slightly younger age, although the distributions still overlap.

As we explained in Section 4.1, MCMC compares the observed photometry to the predicted photometry of each star given the set of stellar cluster parameters for a given step in the MCMC chains. The predicted magnitudes of each star can then be averaged over the course of the chain and compared to the data. This demonstrates how well MCMC models the photometry and indicates the reliability of the fit.

In both panels of Figure 8, we display the CMD of just the WD region of NGC 2477, with the photometry of the WD candidates given by the black points and error bars. In the left panel, we plot the average values of the photometry as predicted by MCMC for the DSED model set, represented by the blue stars. We note that for WD2, the final cluster membership probability from each MCMC run was typically low, often less than 10\%, explaining for the somewhat skewed predicted photometry. Also overplotted is a WD isochrone, simulated with the best fit cluster parameters (by MCMC using the DSED models) listed in Table 6. This figure demonstrates that the photometry data were fit very well and that the combination of parameters found (especially age) by MCMC did an excellent job at fitting the data. The right panel of this plot shows multiple

WD isochrones simulated with the values in Table 6 for all models, for easy comparison of the models in color-magnitude space.

Our final value for the WD age of this cluster comes by taking a weighted average of the ages determined by all three model sets, calculated in the standard way, with weights being inversely proportional to the MS model specific variances. We then calculated two error bars. The first 
represents a quality of the model fit to the data (i.e., internal or within-model error) and is found by taking the square root of the average of the variances for each MS model (i.e., the square of the standard deviations, as listed in Table 6). The second represents the spread among the three different models we used, found by computing the standard deviation of the three model-specific fitted values (i.e., average of the MCMC chains, see Table 6). It is a preliminary measurement of the theoretical uncertainty in the fits, to the degree that these three models span the range in uncertainty in the input physics. (Although they do not capture all of that uncertainty, it is a start in the process of quantifying it.)

With this in mind, our final value for the WD age of NGC 2477 is $1.035 \pm 0.054 \pm 0.087$ Gyr.

\section{Conclusions}

In this paper, we have presented deep HST observations of NGC 2477. We have estimated a WD age of this cluster using traditional techniques of fitting WD isochrones by eye, resulting in a WD age of approximately 1.0 Gyr. In order to achieve higher precision in clusters ages, our group has developed a new algorithm that utilizes Bayesian statistics. By employing an MCMC technique, we are able to sample from the posterior distributions of cluster parameters, specifically age, performing a simultaneous best fit of the free parameters to the photometry. WD ages determined from this method have much higher precision than by simply fitting isochrones by eye. Using the Bayesian algorithm and utilizing different MS evolution timescale models, we have measured the WD age of NGC 2477 to be $1.035 \pm 0.054 \pm 0.087$ Gyr (uncertainties represent the goodness of model fits and discrepancy among models, respectively). This age is consistent with that measured from traditional isochrone fitting (both from MSTO fitting by Kassis et al. 1997, and the initial WD age derived by von Hippel et al. 1995), as we would expect, but our new result is objective, marginalizes over the other cluster parameters, and includes posterior distributions on all parameters. Even with only a few WDs, our technique achieves a $5 \%$ age precision within

a given model, and when marginalizing over three MS stellar evolution models, it achieves better than $9 \%$ precision.

This material is based upon work supported by the National Aeronautics and Space Administration under Grant NAG5-13070 issued through the Office of Space Science, and by the National Science Foundation through Grant AST-0307315, as well as NSF grant DMS-0907522.

\section{REFERENCES}

Baggett, S. et al. 2002, HST WFPC2 Data Handbook v. 4.0, ed. B. Mobasher, Baltimore, STScI Bedin, Luigi R., Salaris, Maurizio, Piotto, Giampaolo, King, Ivan R., Anderson, Jay, Cassisi, Santi, Momany, Yazan 2005, ApJ, 624, L45 
Bedin, L. R., Salaris, M., Piotto, G., Cassisi, S., Milone, A. P., Anderson, J., King, I. R. 2008a, ApJ, 679, L29

Bedin, L.R., King, I.R., Anderson, J., Piotto, G., Salaris, M., Cassisi, S., \& Serenelli, A. 2008b, ApJ, 678, 1279

Bellazzini, M., Ibata, R., Monaco, L., Martin, N., Irwin, M. J., \& Lewis, G.F. 2004, MNRAS, 354, 1263

Bellini, A. et al. 2010, A\&A, 513, 50

Bergeron, P., Wesemael, F., \& Beauchamp, A. 1995, PASP, 107, 1047

Bertin, E. \& Armouts, S. 1996, A\&AS, 117, 393

Carraro, G., Vzquez, R.A., Moitinho, A., \& Baume, G. 2005, ApJ, 630, L153

Chaboyer, B., Demarque, P., \& Sarajedini, A. 1996, ApJ, 459, 558

Claver, C. F. 1995, PhD. Thesis, The University of Texas at Austin

Claver, C. F., Liebert, J., Bergeron, P. 2001, ApJ, 563, 987

DeGennaro, S., von Hippel, T., Jefferys, W.H., Stein, N., van Dyk, D., \& Jeffery, E. 2009, ApJ, 696, 12

Deloye, C. \& Bildsten, L. 2002, ApJ, 580, 1077

Dias, W.S., Assafin, M., Flrio, V., Alessi, B.S., \& Lbero, V. 2006, A\&A, 446, 949

Dolphin, A.E. 2000, PASP, 112, 1397

Dotter, A., Chaboyer, B., Jevremovic, D., Kostov, V., Baron, E., \& Ferguson, J.W. 2008, ApJS, 178, 89

Eigenbrod, A., Mermilliod, J.-C., Claria, J. J., Andersen, J., Mayor, M. 2004, A\&A,423,189

Friel, E.D. \& Janes, K.A. 1993, A\&A, 267, 75

Garcia-Berro, E. et al. 2009, Nature, 465, 194

Girardi, L., Bressan, A., Bertelli, G., \& Chiosi, C. 2000. A\&AS, 141, 371

Hansen, B.M.S., et al. 2007, ApJ, 671, 380

Hansen, B.M.S. 2005, ApJ, 635, 522

Hansen, B.M.S., et al. 2002, ApJ, 574, L155

Hartwick, F.D.A., Hesser, J.E., McClure, R.D. 1972, ApJ, 174, 557

Holtzmann, J.A., Burrows, C.J., Casertano, S., Hester, J.J., Watson, A.M., \& Worthy, G.S. 1995, PASP, 107, 1065

Jeffery, E.J., von Hippel, T., Jefferys, W.H., Winget, D.E., Stein, N., DeGennaro, S. 2007, ApJ, 658,391

Kalirai, J. et al. 2007, ApJ, 671, 748

Kassis, M., Janes, K. A., Friel, E. D., \& Phelps, R. L. 1997, AJ, 113, 1723

Kepler, S.O., Kleinman, S.J., Nitta, A., Koester, D., Castanheira, B., Giovannini, O., Costa, A.F.M., Althaus, L. 2007, MNRAS, 375, 1315

Kilic, M., von Hippel, T., Mullally, F., Reach, W.T., Kuchner, M.J., Winget, D.E., \& Burrows, A. 2006, ApJ, 642, 1051

Knox, R.A., Hawkins, M.R.S., \& Hambly, N.C. 1999, MNRAS, 306, 736

Koekemoer, A.M. et al. 2002, HST Dither Handbook, Version 2.0, Baltimore, STScI 
Leggett, S.K., Ruiz, M.T., \& Bergeron, P. 1998, ApJ, 497, 294

Momany et al. 2001, A\&A, 379, 436

Mestel, L. 1952, MNRAS, 112, 583

Mighell, K.J., \& Rich, R.M. 1995, AJ, 110, 1649

Miller, G. E., \& Scalo, J. M. 1979, ApJS, 41, 513

Montgomery, M.H., Klumpe, E.W., Winget, D.E., \& Wood, M.A. 1999, ApJ, 525, 482

Oswalt, T.D., Smith, J.A., Wood, M.A., \& Hintzen, P. 1996, Nature, 382, 692

Richards, G.T. et al. 2009, ApJS, 180, 67

Richards, G.T. et al. 2006, AJ, 131, 2766

Richer, H.B. 2010, STScI May Symposium

Salaris, M., Weiss, A., \& Percival, S.M. 2004, A\&A, 414, 163

Salaris, M., Garca-Berro, E., Hernanz, M., Isern, J., \& Saumon, D. 2000, ApJ, 544, 1036

van Dyk, D. A., DeGennaro, S., Stein, N., Jefferys, W. H., and von Hippel, T. 2009, The Annals of Applied Statistics, 3, 117

van Loon, J.T., Boyer, M.L., \& McDonald, I. 2008, ApJ, 680, L49

von Hippel, T. 2005, ApJ, 622, 565

von Hippel, T. 2001, in ASP Conf. Ser. 245, ed. T. von Hippel et al., (San Fransisco: ASP), 190

von Hippel, T. \& Gilmore, G. 2000, AJ, 120, 1384

von Hippel, T., Gilmore, G., \& Jones, D.H.P. 1995, MNRAS, 273, L39

von Hippel, T., Gilmore, G., Nial, T., Robinson, D., \& Jones, D.H.P. 1996, AJ, 112, 196

von Hippel, T., Jefferys, W.H., Scott, J., Stein, N., Winget, D.E., DeGennaro, S., Dam, A., \& Jeffery, E. 2006, ApJ, 645, 1436

Weidemann, V. 2000, A\&A, 363, 647

Winget, D. E., Hansen, C. J., Liebert, J., van Horn, H. M., Fontaine, G., Nather, R. E., Kepler, S. O., \& Lamb, D. Q. 1987, ApJ, 315, L77

Wood, M. A. 1992, ApJ, 386, 539

Yi, S. Demarque, P., Kim, Y.-C., Lee, Y.-W., Ree, C.H., Lejeune, T., \& Barnes, S. 2001, ApJS, 136,417 


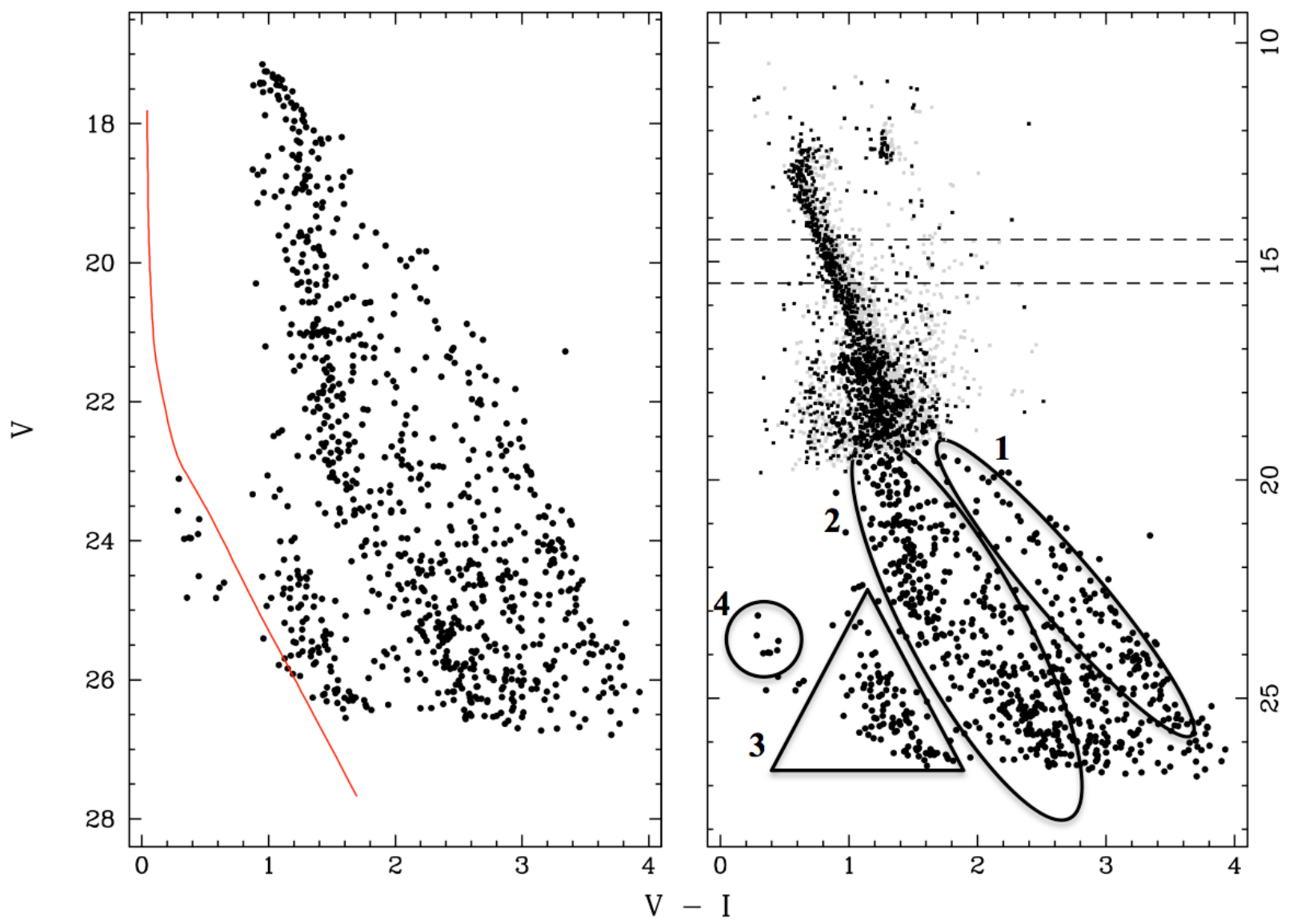

Fig. 1. - Left: CMD of NGC 2477 taken with HST/WFPC2. Only objects with magnitude errors less than 0.1 have been plotted. Overplotted is the cooling track of a $0.6 M_{\odot}$ WD (Wood 1992). Right: Ground-based photometry (small black and gray points) of NGC 2477 combined with HST data (large black points). The numbered regions indicate (1) the cluster MS; (2) a substantial stellar background population; (3) other field stars, image defects, and background galaxies; and (4) the cluster WD sequence. The dashed lines in this panel indicate the portion of the MS used when running MCMC (see Section 4.2). That is, when running MCMC, only the portion of the MS between the dashed lines as well as the cluster WDs candidates are used. The rest of the data are discarded. 


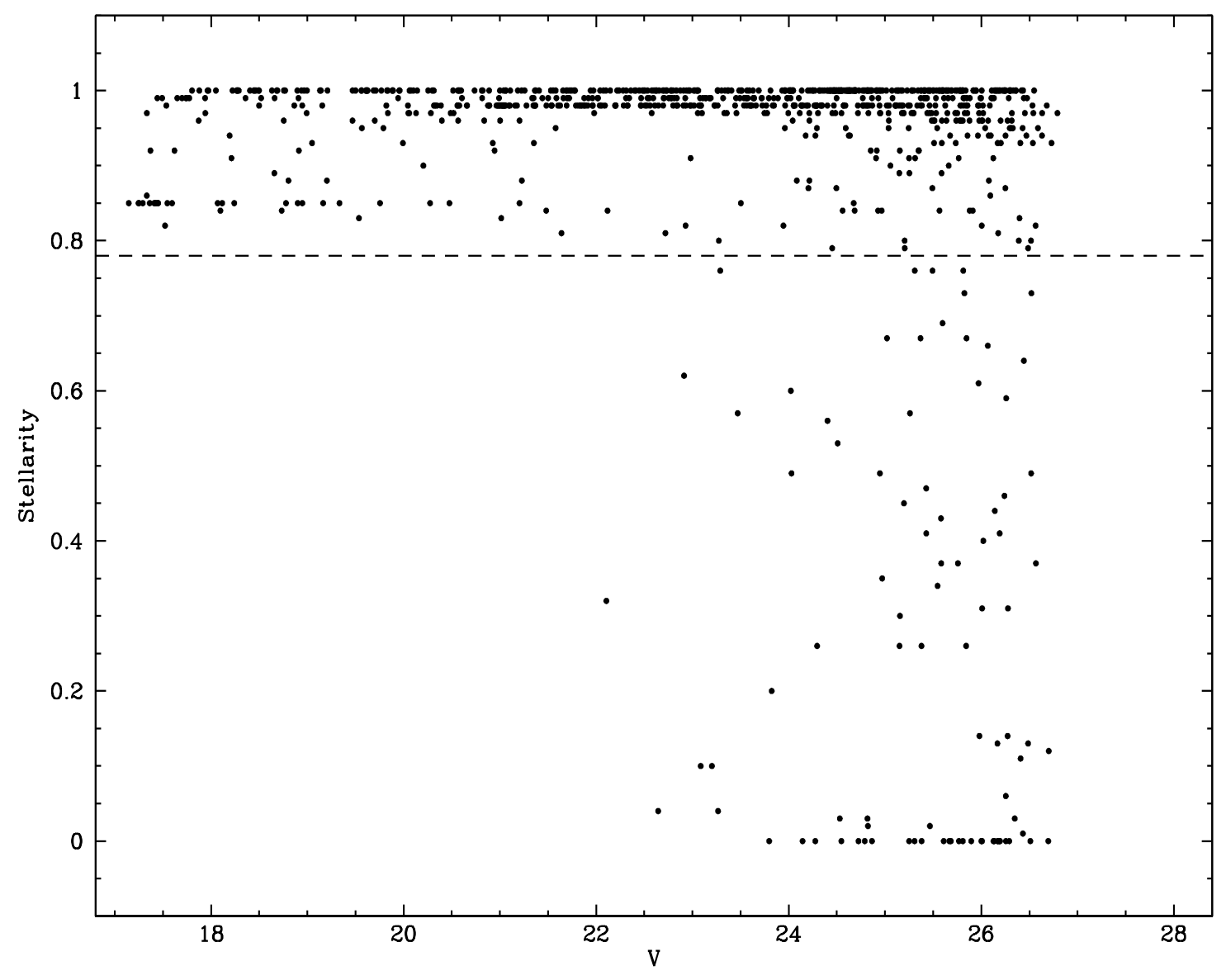

Fig. 2.- Stellarity index vs. V magnitude. Stellarity index ranges from 0 (galaxies) to 1 (stars). Objects with stellarity $\leq 0.78$ (indicated by the dashed line) were automatically rejected from the analysis. 


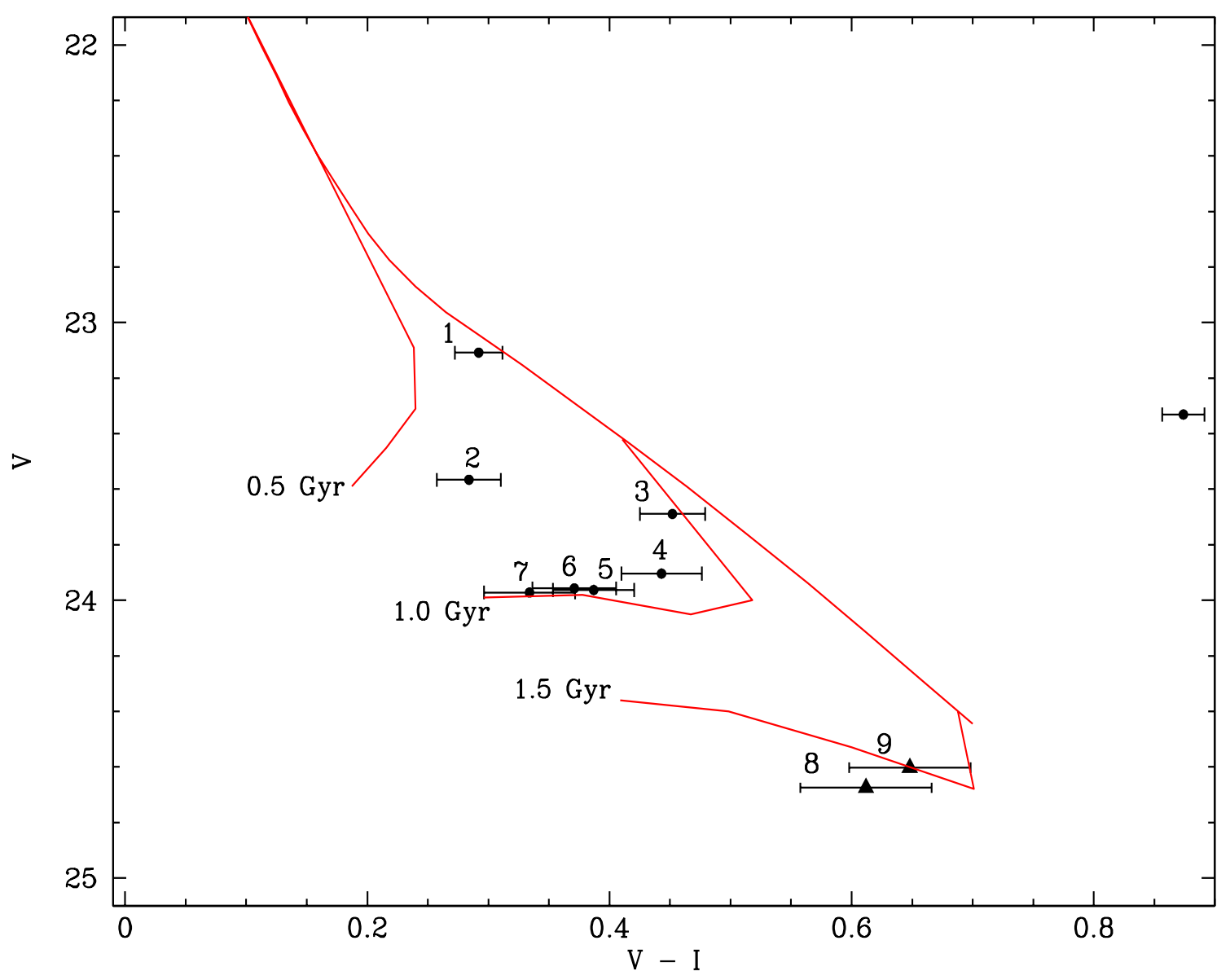

Fig. 3. - CMD of NGC 2477, zoomed on the WD region. Only objects passing the first stellarity cut (Figure 2) are plotted. Each object was then visually inspected on the original images. Objects 1-7 are confirmed to be stellar and we assume them to be the cluster WDs; objects 8 and 9, plotted as triangles, are found to be image defects (see Figure 4) and will be discarded from further analysis. We have overplotted WD isochrones (from Wood 1992) for 0.5, 1.0, and 1.5 Gyr. The best fit to the WDs (i.e., objects 1-7) in this cluster is $1.0 \mathrm{Gyr}$, in good agreement with the MSTO age. 


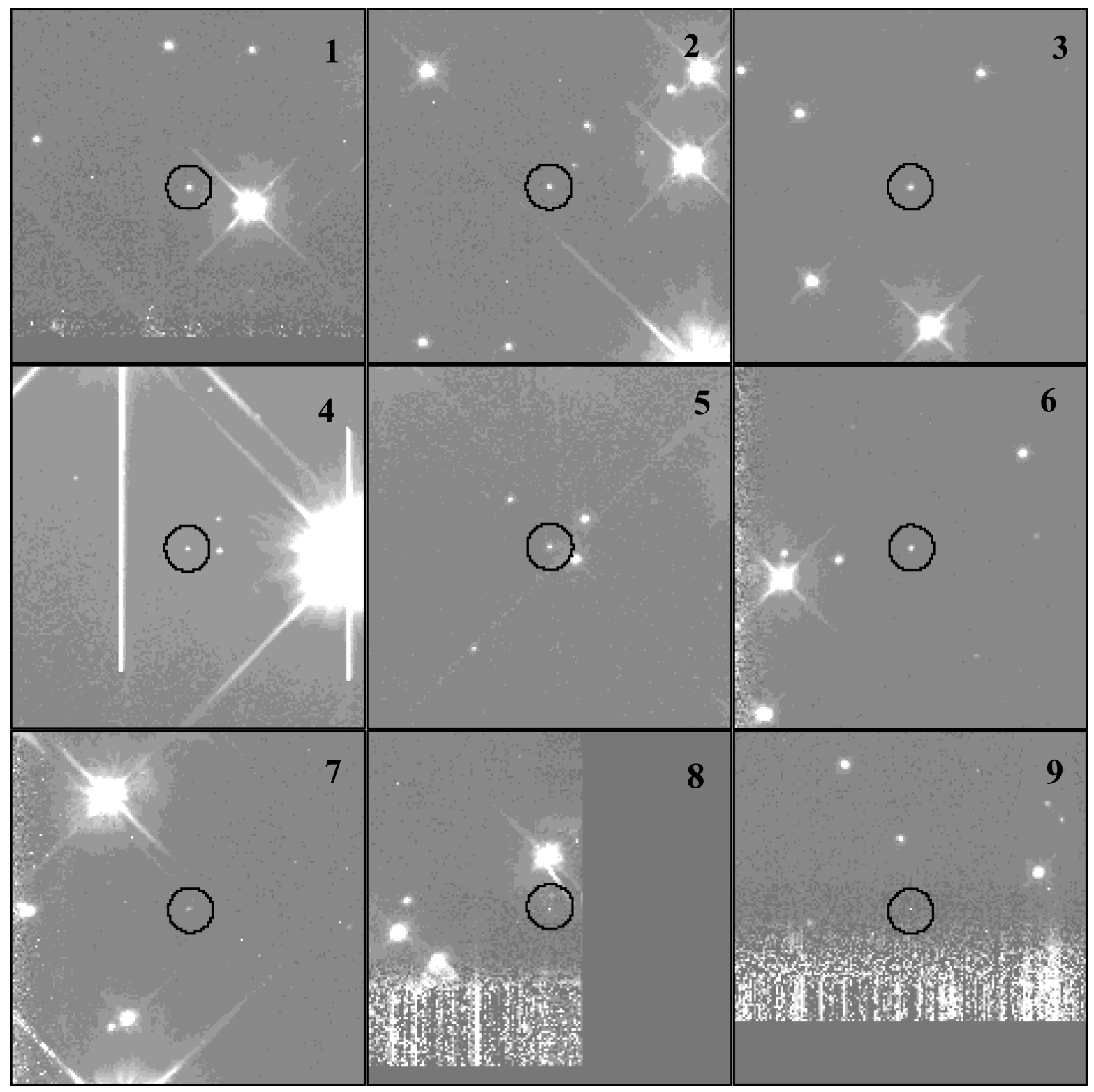

Fig. 4.- Image screenshots of the nine blue objects in the WD region of the CMD with high stellarity (objects 1 through 9, see Figure 3). (We note that we are displaying the imcombined, not drizzled, images. See text for further discussion.) Visual inspection confirms that objects 1 7 are indeed stellar, while 8 and 9 are they are likely image defects, found in the noisy vignetted edge region of the CCD. Because of this, these two objects will be completely discarded for further analysis. 

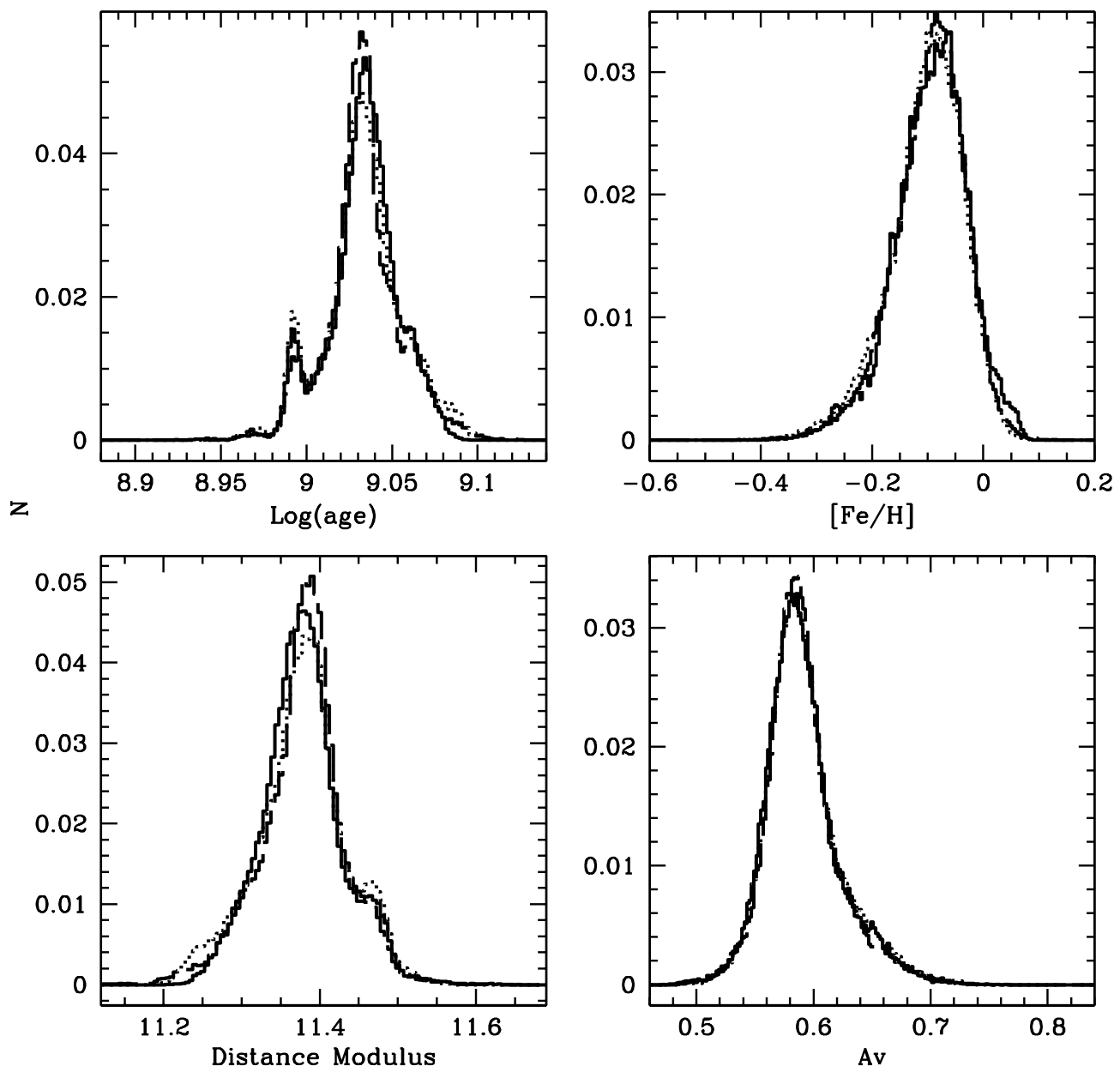

Fig. 5.- Comparison of posterior distributions of cluster parameters given different start values for $\log ($ age $)$, namely $\log ($ age $)=8.9,9.0$, and 9.1, represented by different line styles: solid (8.9), dashed (9.0), and dotted (9.1). We note how consistently MCMC found the posterior distribution, regardless of starting value. This demonstrates the robustness of the technique to the age starting value, and the plausibility of no additional modes in this area of parameter space. 

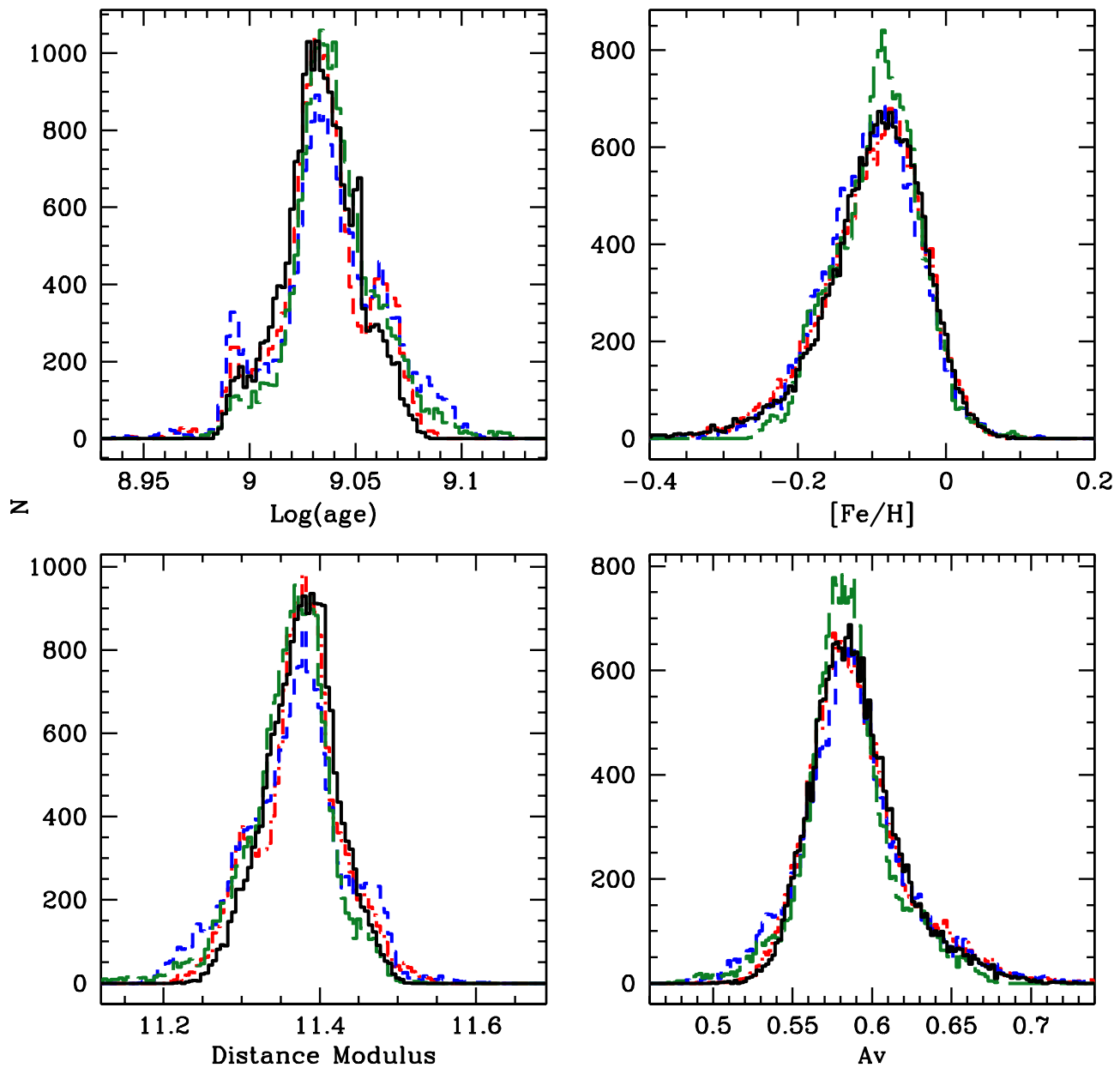

Fig. 6.- Histograms of the sampling histories for the cluster parameters of NGC 2477 using different initial random seeds for each run. The results plotted here used the DSED MS models with a $\log$ (age) starting value of 9.0 (see Section 4.3). Different runs using different random seeds are represented by different colors and line styles. From this it can be seen how consistently MCMC found the location of the posterior distribution for sampling, regardless of the initial random seed. 

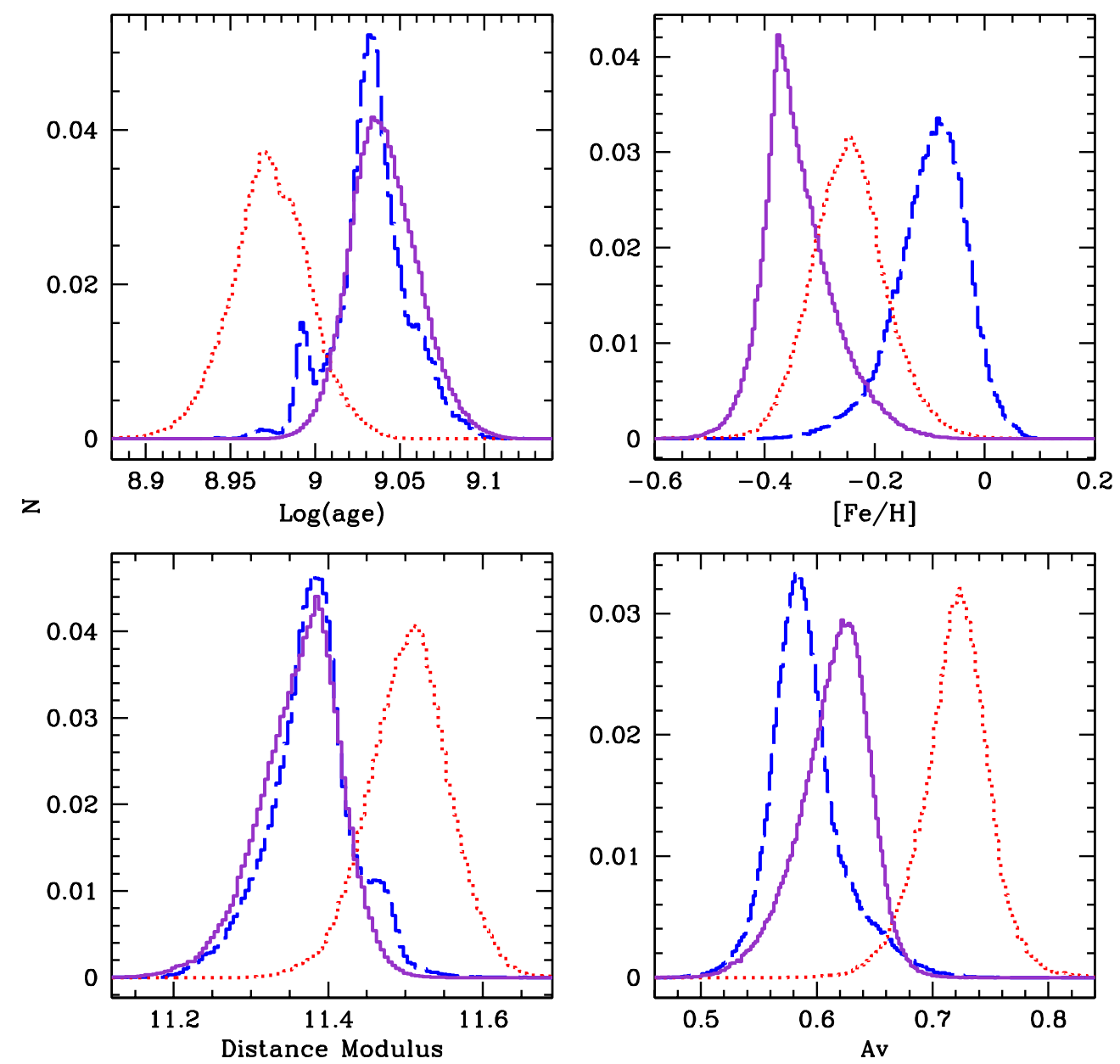

Fig. 7.- Comparison of the combined posterior distributions of cluster parameters given the different MS evolution models. Each posterior distribution is the combination of twelve individual MCMC runs (i.e., three different $\log$ (age) starting values, each run with four different random seeds). The different models are represented by different line styles: solid purple (Girardi et al. 2000), dotted red (Yi et al. 2001), and dashed blue (Dotter et al. 2008). For age, DSED and Girardi models are in the best agreement, while the YY models give a slightly lower age, although the distributions are all overlapping. We have listed the average and standard deviation for each of these distributions in Table 6 , 


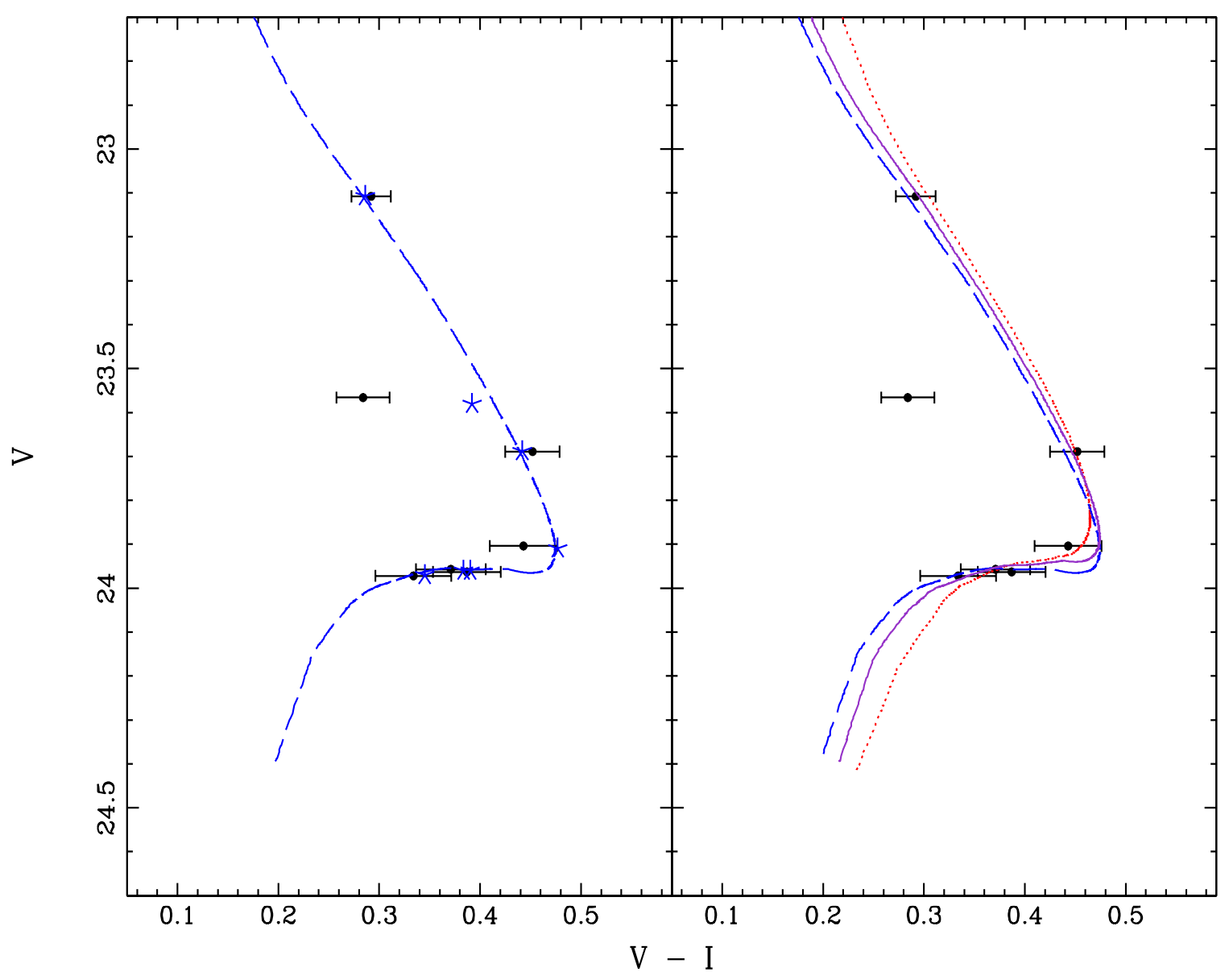

Fig. 8.- The CMD of the WD region of NGC 2477. In both panels, the solid black points are the data. On the left, the blue stars represent the average of the photometry values produced by MCMC (using the DSED models). A WD isochrone simulated with the DSED values in Table 6 is overplotted to demonstrate the quality of the fit to the data. (We also note that for WD2 in this panel, the final cluster membership probability from each MCMC run was typically low, often less than 10\%, which explains for the offset predicted photometry value.) The right panel of this plot shows multiple WD isochrones simulated with the appropriate values for that model, listed in Table 6, for easy comparison of the models in color-magnitude space. Color and line styles are consistent with those used in Figure 7 . 


\begin{tabular}{ccccc}
\hline Age (Gyr) & $(m-M)_{V}$ & $A_{V}^{a}$ & {$[\mathrm{Fe} / \mathrm{H}]$} & Source \\
\hline 1.0 & 11.43 & 0.93 & - & 1 \\
1.0 & 11.45 & 0.713 & 0.00 & 2 \\
$1.0^{b}$ & - & - & - & 3 \\
1.0 & - & - & -0.14 & 4 \\
1.3 & 11.60 & 0.93 & -0.05 & 5 \\
1.5 & 11.48 & 0.868 & - & 6 \\
\hline
\end{tabular}

${ }^{1}$ Kassis et al. 1997, ${ }^{2}$ Salaris et al. 2004, ${ }^{3}$ von Hippel, Gilmore, \& Jones, 1995, ${ }^{4}$ Eigenbrod et al. 2004, ${ }^{5}$ Friel \& Janes 1993, ${ }^{6}$ Hartwick, et al. $1972 ;{ }^{a}$ Average value; ${ }^{b}$ White dwarf age

Table 1: Cluster parameters from the literature for NGC 2477.

\begin{tabular}{ccc}
\hline Field & RA(2000) & $\operatorname{Dec}(2000)$ \\
\hline 1 & $07: 52: 16.81$ & $-38^{\circ} 35^{\prime} 40^{\prime \prime}$ \\
2 & $07: 52: 26.20$ & $-38^{\circ} 34^{\prime} 40^{\prime \prime}$ \\
\hline
\end{tabular}

Table 2: Coordinates of observed fields for NGC 2477 with HST/WFPC2.

\begin{tabular}{ccccc}
\hline $\begin{array}{c}\text { Date } \\
\text { Observed }\end{array}$ & Filter & $\begin{array}{c}\text { Exposure } \\
\text { Time (s) }\end{array}$ & Field & Cycle \\
\hline 1994 Mar 18 & F555W & $8 \times 400$ & 1 & 4 \\
1994 Mar 18 & F814W & $9 \times 400$ & 1 & 4 \\
1996 Mar 25 & F555W & $8 \times 500$ & 1 & 6 \\
1997 Mar 17 & F555W & $12 \times 500$ & 2 & 6 \\
1997 Mar 17 & F814W & $8 \times 500$ & 2 & 6 \\
\hline
\end{tabular}

Table 3: Summary of observations for NGC 2477 with HST/WFPC2.

\begin{tabular}{ccccccc}
\hline ID & $V$ & $\sigma_{V}$ & $V-I$ & $\sigma_{V-I}$ & R.A. & Dec. \\
\hline WD1 & 23.108 & 0.008 & 0.292 & 0.020 & $07: 52: 22.6$ & $-38^{\circ} 35^{\prime} 40.9^{\prime \prime}$ \\
WD2 & 23.566 & 0.011 & 0.284 & 0.026 & $07: 52: 28.3$ & $-38^{\circ} 34^{\prime} 54.3^{\prime \prime}$ \\
WD3 & 23.689 & 0.010 & 0.452 & 0.027 & $07: 52: 22.9$ & $-38^{\circ} 35^{\prime} 54.4^{\prime \prime}$ \\
WD4 & 23.904 & 0.012 & 0.443 & 0.033 & $07: 52: 12.5$ & $-38^{\circ} 36^{\prime} 10.2^{\prime \prime}$ \\
WD5 & 23.963 & 0.015 & 0.387 & 0.034 & $07: 52: 25.6$ & $-38^{\circ} 35^{\prime} 21.7^{\prime \prime}$ \\
WD6 & 23.957 & 0.015 & 0.371 & 0.034 & $07: 52: 23.3$ & $-38^{\circ} 34^{\prime} 32.1^{\prime \prime}$ \\
WD7 & 23.972 & 0.016 & 0.334 & 0.038 & $07: 52: 21.8$ & $-38^{\circ} 34^{\prime} 46.9^{\prime \prime}$ \\
\hline
\end{tabular}

Table 4: Table of WD candidates in NGC 2477, including photometry and coordinate information. Numbering is consistent with Figure 3. We note that only confirmed stellar objects are listed. 


\begin{tabular}{lcc}
\hline \multicolumn{1}{c}{ Cluster } & Mean & \\
Parameter & Value & $\sigma$ \\
\hline$[F e / H]$ & -0.10 & 0.30 \\
$(m-M)_{V}$ & 11.460 & 0.22 \\
$\mathrm{~A}_{V}$ & 0.750 & 0.10 \\
\hline
\end{tabular}

Table 5: Priors used by the Bayesian algorithm. Mean values are within literature values for these parameters (see Table 10) and $\sigma$ values are representative of conservative uncertainty for these quantities. The mean values of these distributions were used as the starting value for MCMC, as mentioned in Section 4.3 .

\begin{tabular}{lcccccc}
\hline \multicolumn{1}{c}{ Cluster } & DSED & & YY & \multicolumn{3}{c}{ Girardi } \\
Parameter & Mean & $\sigma_{D S E D}$ & Mean & $\sigma_{Y Y}$ & Mean & $\sigma_{G}$ \\
\hline Age $(\mathrm{Gyr})$ & 1.08 & 0.06 & 0.94 & 0.05 & 1.10 & 0.05 \\
{$[\mathrm{Fe} / \mathrm{H}]$} & -0.098 & 0.05 & -0.246 & 0.07 & -0.338 & 0.07 \\
$(m-M)_{V}$ & 11.378 & 0.07 & 11.504 & 0.06 & 11.363 & 0.06 \\
$\mathrm{~A}_{V}$ & 0.591 & 0.03 & 0.720 & 0.03 & 0.615 & 0.03 \\
\hline
\end{tabular}

Table 6: Mean and standard deviation of the posterior distributions of cluster parameters of NGC 2477 from WD plus MS photometry. We note that the small sigma on $A_{V}$ should not be taken to imply that the cluster does not exhibit differential reddening (as was mentioned in Section 1); rather, our model does not incorporate differential reddening at this time. The sigma here is an error on the mean reddening. 